\title{
Surge amica mea. Zum Verhältnis von Religion und Humor am Beispiel der Konjunktion von Natur und Geschichte bei Johann Georg Hamann und Jean Paul
}

Für Tamara Ehrenbold

\author{
Mystiker ist, wer nicht aufhören kann zu wandern \\ und wer in der Gewissheit dessen, was ihm fehlt \\ von jedem Ort und von jedem Objekt weiss: Das ist es nicht \\ Michel de Certeau, Mystische Fabel
}

\section{Methodische Reflexion: Rehabilitation der Enthymeme als philosophischer Humor}

(Sinn, Amir, Certeau)

Als Erfinder ihrer eigenen Wege durch den undurchdringlich gewordenen Dschungel von Rationalisierungsbemühungen am Ende der Frühen Neuzeit versuchen Hamann und Jean Paul aus „dem schwächsten von zwei Argumenten das stärkste "1 $\mathrm{zu}$ machen, denn lebendiger und interessanter als das offizielle philosophische Geklapper mit Begriffen sind ihnen Situationen, die zweifelhaft sind und der Interpretation bedürfen. Die Position des Schwächsten aber ist in dieser Zeit nicht allein das Selbstverständnis der Individuen als soziale wie moralische Akteure, ${ }^{2}$ sondern eben der von Aristoteles, aber auch schon von Platon $^{3}$ kritisierte sophistische Status scheinbarer Enthymeme als solcher. Beide aber sind aufs Engste miteinander verflochten: Enthymeme verkörpern das Prinzip intellektueller Kreativität, das Individuen benötigen, wenn ihnen rein äusserliche Normen und tradierte Begriffe zur Handlungsorientierung nicht mehr genügen.

Hamann und Jean Paul versuchen in je unterschiedlicher Weise die Position des Schwächsten, nämlich der Enthymeme, so stark wie möglich zu machen. Nur von diesem theoretischen Impuls aus sind ihre Bemerkungen zu Konjunktion

\footnotetext{
1 Aristoteles: Rhetorik, II, Kap. 24, 1402a.

2 Das Band der Gesellschaft. Verbindlichkeitsdiskurse im 18. Jahrhundert. Hg. von Simon Bunke, Katerina Mihaylova und Daniela Ringkamp. Tübingen 2015.

3 Platon: Phaidros, 273b-c.
} 
von Natur und Geschichte verständlich. Denn diese Konjunktion ist bereits enthymematisch verfasst: Ein gemeinsamer Grund zweier Begriffe muss gesucht werden, die sich konträr oder sogar kontradiktorisch zueinander verhalten.

Im Verlauf der Arbeit hat das ursprüngliche Vorhaben, durch eine argumentanalytische Klärung des Status von Enthymemen die Konjunktion von Natur und Geschichte bei Hamann und Jean Paul präziser als zuvor zu erfassen, jedoch eine leichte Veränderung erfahren. Unvorhersehbar war für mich die Einsicht, dass Natur und Geschichte bei Hamann und Jean Paul mit einem anderen enthymematischen Zusammenhang, dem von Religion und Humor, in einer zum Teil unlesbaren Querverbindung stehen. Auch wenn die Konjunktion von Natur und Geschichte aus dem Wortschatz offizieller Theologie und Philosophie gebildet wird und dem akademischen Diskurs unterworfen bleibt, so verweist sie doch auf andere Interessen und Wünsche, die sich der Verbindung von Religion und Humor verdanken.

Über diesen Bezug ist so gut wie nichts bekannt, da sich der akademische Diskurs darauf beschränkt, jene Teilbegriffe zu klären, zu differenzieren und zu historisieren, aus denen die Konjunktion von Natur und Geschichte zwar zusammengesetzt ist, auf die sich aber nicht reduzieren lässt. Dieser Diskurs erfasst das historische Material, aber nicht seine humoristische Form, er bezieht sich auf die begriffsgeschichtlich verwendeten Elemente und nicht auf den Hamann und Jean Paul gemeinsamen humoristischen ,Geist', der sich aus der Bastelei mit Enthymemen und topoi ergibt, einer Kombinatorik, die sich zwar rekonstruieren lässt, darum aber noch nicht ihren eigentümlichen Sinn erfasst. Indem die historische und philosophische Forschung die herumvagabundierenden, scheinbar irrationalistischen Zeichenspielereien dieser Autoren in ihre selbstgegebenen Begriffe zerlegt, entmythologisiert sie zwar Tradition, findet aber dafür auch nur das wieder, was sie bereits wusste. Wissenschaft ist ein selbstreproduzierendes System, dass Divergenz und Ambiguität eliminiert. In ihrer analytischen Kompetenz entgeht ihr das, was sie zu bestimmen glaubt. Ich bin mir daher im Klaren, dass ich mich mit dem vorliegenden Versuch in ein szientifisches Abseits stelle. Nicht nur gerät der folgende Beitrag aufgrund seiner Darstellungskomplexität in den performativen Widerspruch, das Ziel zu verfehlen, das er primär anvisiert, nämlich eine bessere Rezeptionssituation für Hamann und Jean Paul im kulturellen Bewusstsein herzustellen. Er mutet Unberechenbarkeit und Wissenschaftskritik zu.

Aus der hier skizzierten Verlegenheit, ein grundsätzliches Thema nicht nur darzustellen, sondern allererst darstellbar zu machen, helfen jedoch mindestens folgende drei Hebel der Theorie: Erstens kann man unabhängig von den Kontexten von Natur und Geschichte wie Religion die von Hamann und Jean Paul 
gebrauchte enthymematische Form formallogisch rehabilitieren, indem man Enthymeme durch folgende drei besonders einfache Axiome rekonstruiert ${ }^{4}$ :

A1: Zwischen zwei verschiedenen topoi $t_{1}$ tm gibt es genau einen topos $t_{n}$, der beide verknüpft.

A2: Es gibt drei verschiedene topoi, die nicht miteinander verknüpft sind.

A3: $\mathrm{Zu}$ einer Verknüpfung $\mathrm{v}$ und einem nichtverknüpften topos $\mathrm{t}$ gibt es genau eine Verknüpfung v' mit $\mathrm{t}$, die keinen topos mit $\mathrm{v}$ gemeinsam hat

Die Worte „topoi“ und „Verknüpfung“ sind zwar der Bildungssprache entnommen. Sie werden aber in rein formale Relationen überführt. Wesentlich in diesem Modell ist, dass nicht unendlich viele topo $i$ verlangt werden. Es handelt sich um ein flexibles Argumentationsmodell in seiner Minimalform mit nur einem Enthymem als mittlerem topos, so dass es einerseits der Mehrwertigkeit faktischer und literarischer Argumentationen gerecht werden kann, andererseits nicht in Undarstellbarkeit versinkt. Diese Axiomatisierung hat folgenden Vorteil: Das Enthymem verschwindet nicht mehr, wie z. B. bei Peirce und Eco, aber auch dekonstruktivistischen Varianten in Modellen unendlicher semiosis. Vielmehr erscheint hier die Unabhängigkeit eines Enthymems als Basis dieser semiosis, deren überfordernder Wirbel unterbrochen wird, um sich in einer möglichst einfachen Form anzunähern.

Ein Enthymem wird danach durch die einfachste Kombination lauten: A1 und A2 seien erfüllt, nicht jedoch A3. Die Topologie besteht dann aus drei nicht miteinander verbundenen topoi $\mathrm{t}_{1}, \mathrm{t}_{2}$ und $\mathrm{t}_{3}$. $\left(\mathrm{t}_{1}, \mathrm{t}_{2}\right)$ und $\left(\mathrm{t}_{2}, \mathrm{t}_{3}\right)$ sind dann miteinander durch das Enthymem $t_{2}$ verknüpft:

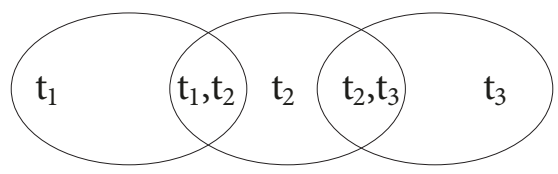

Die Leserin soll nicht mit Logeleien gequält werden. Wichtig ist lediglich, dass diese dreiaxiomatige Topologie argumentative Zusammenhänge überschaubar und überprüfbar macht und daher eine materiale Logik der Erfahrung modellieren kann: Man fragt lediglich nach Implikationsverhältnissen von topoi, die auf ein topologisches, quantifizierbares Modell abgebildet werden, um so Ar-

4 Zum weiteren Theoriekontext: Christian Sinn: Dichten und Denken. Entwurf einer Grundlegung der Entdeckungslogik in den exakten und ,schönen' Wissenschaften. Aachen 2001, S. 24-47. Ich habe die oben skizzierte Topologie auf die Beispiele von Sebastian Brants Narrenschiff und Erasmus von Rotterdams Lob der Torheit angewendet und textanalytisch nachgewiesen, wie sich ihre Form von Argumentation als Konvergenz grundsätzlich von Argumentation als Kohärenz (Platon, Aristoteles) unterscheidet. 
gumentationen überprüfen zu können. Bei komplexen Argumentationen hat dies den Vorteil, dass auf visuelle Weise überprüft werden kann, ob sie gültig sind oder qua visueller Homologie auf eine bereits bekannte, fehlerhafte Argumentation zurückgeführt werden können.

Es sprengte den Rahmen dieser Darstellung zu zeigen, wie Theologien wie z. B. Lulls Kombinatorik sich selbst widersprechen, wenn in ihnen Gott als gut, gross, mächtig und weise bezeichnet wird, weil man sie isomorph auf absurde Argumentationen abbilden kann. Hier interessiert allein das Faktum, dass die offizielle Philosophie (Platon, Aristoteles) selbst sophistisch verfährt, wenn sie Enthymeme lediglich als sophistische Stärkung der schwächsten Position kennzeichnet. Entscheidend ist für den vorliegenden Zusammenhang erstens, dass nicht die Enthymeme, sondern die durch sie erzeugten Implikationsverhältnisse zur Diskussion stehen und zweitens, dass Hamann und Jean Paul in ihren Texten durch solche Implikationsverdichtungen eine materiale Logik der Erfahrung inszenieren. Darin besteht jenseits vergeblicher philosophiehistorischer Verortungsversuche ihre Gemeinsamkeit wie ihr Humor und daher ist das hier vorgeschlagene Modell methodisch signifikant.

Zum anderen liegt inhaltlich gesehen hinsichtlich der Konjunktion von Natur und Geschichte im Zusammenhang von Religion und Humor bereits ein Beitrag von Lydia B. Amir vor. In ihrer bedeutenden Studie Humor and the good life in modern philosophy, ${ }^{5}$ verweist sie auf Shaftesbury als Ausgangspunkt von Hamann und Jean Paul. ${ }^{6}$ Angesichts der deutschen Rezeptionssituation, die die zentrale Bedeutung Shaftesburys für Hamann, Wieland, Jean Paul, Friedrich Schlegel u. a. und nicht zuletzt als Grenzscheide zu der von Lessing und Goethe dominierten Inlandgermanistik schlicht nicht wahrnimmt, ist der Beitrag von Amir ausgesprochen wichtig und hilfreich. Amir zeigt, wie Shaftesbury, Hamann und Kierkegaard als überzeugte Christen für ihre Kritik am religiösen Establishment Humor, Ironie und Witz einzusetzen verstehen. Es ist jene Schnelligkeit rhetorischer Bewegungen, die auch bei Jean Paul die Organisation des durch Philosophie und Theologie etablierten Raumes von Natur und Geschichte verändert. Sie aber wird als intellektueller coup nur dann möglich, wenn sie auf die Option von Humor und Religion verweist, mit anderen Worten, die Konjunktion von Natur und Geschichte wird erst durch diese Option möglich. Indem Amir die epistemologischen, ethischen und religiösen Funktionen dieses ursprünglich christlichen Humors untersucht, deutet sie jene ,säkulare` Variante eines philosophischen Humors an, der auf den Widerstand setzt, den kontin-

5 Lydia Amir: Humor and the Good Life in Modern Philosophy. Shaftesbury, Hamann, Kierkegaard. New York 2014, S. 89-99.

6 Ebd., S. 3, 8, 86, 209, 214-217, 219, 279, 299, 320, 327. 
genzerfahrene Ironie durch solidarisches Handeln dem Verschleiss philosophischer Systembildung entgegenhält: ${ }^{7}$

The outcome of this study is the insight that humour, when used to further the philosophic ideals of self-knowledge, truth, rationality, freedom, virtue, happiness, and wisdom, can be one oft he most useful tools available to a philosopher. ${ }^{8}$

Ist jedoch der theoretische Bezugspunkt Kierkegaards nicht nur auf Hamann, sondern ebenso auf Jean Paul fokussiert, ${ }^{9}$ dann ist nicht nur eine bisher verborgen gebliebene Gemeinsamkeit zwischen Hamann und Jean Paul erkennbar, sondern auch dass diese Gemeinsamkeit in der Auffassung von Humor als Inkongruenzerfahrung zwischen endlichem Subjekt und Gott besteht, mithin religiös fundiert ist. Wichtig ist, dass dieser religiöse Humor die Spannung nicht etwa in Gelächter auflöst, sondern perpetuiert und deshalb zugleich in Differenz zu Erlösungslehren der Religion wie quietistisch orientierten Philosophien steht. Diese Auflösungen gehen zu Lasten entweder der Subjekte oder Gottes oder der Vernunft. ${ }^{10}$ Die Konjunktion von Religion und Humor bei Hamann, Jean Paul und dann Kierkegaard ist keine Identität, sondern eine in sich gespannte Differenz, die einen Vertrag mit der Vernunft erfordert und den Irrationalismus der ,Schwärmerei` verhindert:

Shaftesburean humour is the sign of the awareness of finitude. Humour is the acceptance of an inward measure, of an equilibrium that welcomes the inward truth of every situation, even if and maybe because, understanding does not always comprehend the why and how. Humour, for Shaftesbury, is thus the positive expression of the awareness of our limits, both as intelligent and sensible beings. ${ }^{11}$

So ist es nur konsequent, wenn sich diese Akzeptanz von Endlichkeit durch die Vernunft zu einer Akzeptanz ihrer eigenen Kontingenz erweitern muss, - das ist die gemeinsame Pointe Hamanns und Jean Pauls und ihre gleichsam rationale Rechtfertigung von Religion im Zeichen des Humors als Kontingenzakzeptanz:

Weil unsere Vernunft blos aus den äusseren Verhältnissen sichtbarer, sinnlicher, unstätiger Dinge den Stoff ihrer Begriffe schöpft, um selbige nach der Form ihrer innern Natur selbst zu bilden, und zu ihrem Genuß oder Gebrauch anzuwenden: so liegt der Grund der Religion in unserer ganzen Existenz und außer der Sphäre unserer Erkenntniskräfte, welche alle zusammengenommen, den zufälligsten und abstractesten modum unserer Existenz ausmachen. ${ }^{12}$

7 Richard Rorty: Contingency, irony, and solidarity. Cambridge 1989.

8 Amir: Humor and the Good Life (wie Anm. 5), S. 3.

9 Ebd., S. 320.

10 Ebd., S. 9.

11 Ebd., S. 86.

12 N III, 191. 
Die Kontingenz unserer Vernunft sowie das „unendliche[n] Missverhältniss[e] des Menschen zu Gott“, ${ }^{13}$ aber auch das ähnliche „Misverhältnis des Menschen zum Menschen “14 begründen den religiösen Humor und reorganisieren den philosophischen Vernunftraum als Selbstkritik.

Ein weiterer von Amir genannter Bezugspunkt von Hamann und Jean Paul, Giordano Bruno, ${ }^{15}$ präzisiert die zweite Zielrichtung dieser Untersuchung, die Konjunktion von Natur und Geschichte, die im zweiten Teil vertieft werden wird. Die metaphysische Doktrin der coincidentia oppositorum bei Bruno wird bei Hamann, aber auch Jean Paul weniger zur Denk- denn zur Stilfigur der Katachrese, die mit der theologischen Doktrin der Knechtsgestalt Christi assoziiert ist. Die humoristische Katachrese erfüllt im Unterschied zu ironischen Stilfiguren, die über das Ende aller menschlichen Projekte angesichts des Todes triumphieren, eine positive Funktion: sie befreit Menschen qua der absurden Relation zwischen göttlicher und menschlicher Vernunft aus enger Endlichkeit und führt sie zur Akzeptanz paradoxer Wahrheiten, deren ,paradoxeste' in der Inkarnation besteht. Ein solcher Humor kann als Weg zur Erlösung angesehen werden. ${ }^{16}$ Diesen Aspekt, auf den der Titel des Beitrags anspielt, spitzt der dritte Teil im Hinblick auf moderne und szientifische Kontexte zu.

Verschiedene theoretische, aber auch philologische Kreuz-Züge werden es drittens ermöglichen, die unterschiedlichen rhetorischen Taktiken von Hamann und Jean Paul im Feld von Natur und Geschichte als gemeinsame Strategie gegen theologische und philosophische Humorlosigkeit besser zu charakterisieren. Das gilt besonders für jene Figuren und Wendungen, die der in spannungsvoller Distanz zu seinem Orden stehende Jesuit Michel de Certeau in seiner Kunst des Handelns weniger analysiert als theoretische Performanz elegant inszeniert. ${ }^{17}$ Certeau führt Taktiken, Strategien, Poetiken und Rhetoriken, Tricks, Finten und Listen von modernen Konsumenten, jedoch im Kontext uralter historischer Traditionen, geradewegs auf die metis der Natur zurück. ${ }^{18}$ Streifen Certeaus Taktiken den Zusammenhang von Humor und Religion in wiederum humoristischer Weise ${ }^{19}$, so ist die Homologie zu Natur und Geschichte nicht verwun-

$13 \mathrm{~N}$ III, 312.

$14 \mathrm{~N}$ III, 313.

15 Amir: Humor and the Good Life (wie Anm.5), S. 96.

16 Dass dieser Weg nun ausgerechnet für eine Theorie taugt, belegen im Kontext von Religion und Jean Paul Moritz Lazarus: Der Humor als psychologisches Phänomen. In: Ders.: Das Leben der Seele in Monographien über seine Erscheinungen und Gesetze. Bd. I. 3. Ausg., Berlin 1917, S. 29-320; Max Eastman: The Sense of Humour. New York 1922, bes. S. 132, 164-177 und 215.

17 Michel de Certeau: Kunst des Handelns (1980). Aus dem Französischen übersetzt von Ronald Voullié, Berlin 1988.

18 Ebd., S. 12 und 94.

19 Ebd., bes. S. 40, 90, 131, 151, 158, 162, 168, 315-317 und 325. 
derlich, die in Mystische Fabel explizit als Übertragung rhetorischer Tropen auf einen theologischen Gebrauch analysiert wird. ${ }^{20}$ Der Zusammenhang von Natur und Geschichte muss in der mystischen Tradition schon allein deshalb in den von Humor und Religion überführt werden, weil das Johanneische Wort des Lebens nicht allein als historisch aufgefasst wird. Es ist ein leibhaftig gesehenes und berührtes Faktum in der Natur, das als neue Natur erfahren wird. Hierin liegt erst die Begründung dafür, dass sich an diese neue Natur glauben lässt, weil auch von ihr gesagt und geschrieben wurde. ,Humoristisch' kann man diese Verschiebung geschichtlicher Dokumente, die das Wort als lebendige Natur, als incarnatum bezeugen, auf die jeweilige Gegenwart von Religion deshalb nennen, weil es zu einem beständigen Verlangen als Warten auf God[ot] und nicht zuletzt zur Produktion von Texten über dieses Warten führt. ${ }^{21}$ Gerade angesichts der merkwürdigen Tatsache, dass ausgerechnet ein so kluger Geist wie Cusanus den Zeitpunkt der Auferstehung in seiner Coniectura de ultimis diebus (1446) falsch berechnete: In diesem Warten auf God oder Godot sind sich, sei es als gläubige Gewissheit, sei es als Nihilismus, Christen und säkulare Geister gleich.

In diesem Warten durchbrechen Religion und Humor den Kreislauf des Gewöhnlichen, und vielleicht besteht darin ihre einzige Gemeinsamkeit. Doch ist diese nicht nur hinreichend, einen neuen Blick auf die andere, zumeist bestrittene Gemeinsamkeit zwischen Hamann und Jean Paul zu werfen; wichtiger ist gegenwärtig die Einsicht, dass Religion und Humor sich nicht grundsätzlich ausschliessen müssen. Im Rückblick auf beide Autoren kann sich solche Einsicht entwickeln, für die für unsere Kultur vielleicht Kierkegaard der letzte glaubwürdige Zeuge ist. ${ }^{22}$ Allerdings verfügt, wer für beide Gemeinsamkeiten zu argumentieren versucht, für die zwischen Hamann und Jean Paul und noch mehr für die zwischen Religion und Humor, allenfalls über die Stimme eines Predigers in der Wüste. Das ist zwar nun seit den Zeiten Jesajas immer schon die Aufgabe von Religion gewesen: Die Unmöglichkeit, Heil in eine heillose Welt zu bringen, gleichwohl zu wagen. Dazu braucht es Mut und ohne Humor ist solches Wagnis schwerlich möglich. Angemessener scheint freilich die humoristischen Absage an Heilserwartungen jeglicher Couleur: „So sinkt mir der Mut, vor meinen Mitmenschen als Prophet aufzustehen, und ich beuge mich ihrem Vorwurf, daß ich ihnen keinen Trost zu bringen weiß, denn das verlangen sie im Grunde alle,

20 Michel de Certeau: Mystische Fabel. 16. bis 17. Jahrhundert (1982). Aus dem Französischen von Michael Lauble. Mit einem Nachwort von Daniel Bogner. Berlin 2010, bes. S. 143, 299, $474,488,509$ f., $520,528$.

21 Martha Nussbaum: Narrative Gefühle. Becketts Genealogie der Liebe. In: Falsche Gegensätze. Zeitgenössische Positionen zur philosophischen Ästhetik. Hg. von Andrea Kern und Ruth Sonderegger. Frankfurt a. M. 2002, S. 286-329.

22 Amir: Humor and the Good Life (wie Anm.5), S. 101-208. 
die wildesten Revolutionäre nicht weniger leidenschaftlich als die bravsten Frommgläubigen. “23

Erst von diesem freudianischen Punkt aus, über den Verlust, Trost zu spenden, lachen zu können, gesteigert noch zum Lachen über den eigenen Humorverlust, wird man mit Hamann und Jean Paul ins Gespräch und zu jenem Lachen kommen, „worin noch ein Schmerz und eine Größe ist. “24 Doch warum überhaupt mit diesen Toten sprechen? Deutlicher als Stephen Greenblatt ${ }^{25}$ begründet Michel de Certeau die Notwendigkeit der Philologie als Totengespräch, die sie zugleich vom Vorwurf der Nekromantik oder gar der Nekrophilie freispricht:

Einerseits hat das Schreiben [der Geschichte durch die Geschichtswissenschaft] die Funktion eines Bestattungsritus im ethnologischen und quasireligiösen Sinne des Wortes; es treibt den Tod aus, indem es ihn in den Diskurs einbindet. Andererseits besitzt es symbolische Funktion; es ermöglicht einer Gesellschaft, sich zu verorten, indem sie sich durch Sprache eine Vergangenheit gibt, und auf diese Weise öffnet es der Gegenwart einen eigenen Raum: eine Vergangenheit zu kennzeichnen bedeutet, den Toten einen Platz einzuräumen, aber auch den Raum der Möglichkeiten neuzuverteilen, negativ zu bestimmen, was getan werden muß, und folglich die Narrativität, die den Toten begräbt, als Mittel zu benutzen, den Lebenden einen Platz zu sichern. Das Unterbringen der Abwesenden ist die Kehrseite einer Normativität, die auf den lebenden Leser zielt und eine didaktische Beziehung zwischen Sender und Empfänger herstellt. ${ }^{26}$

Soll den Lebenden qua narrativer Bestattung ein Platz gesichert werden, so sind angesichts der übermächtigen Notwendigkeit des Todes Trostworte im Allgemeinen vergeblich. Der Tod lässt sich durch Worte nicht täuschen und die List eines salto natale bleibt daher oft das letzte Mittel, ihm zu begegnen, $\mathrm{zu}$ dem denn auch Certeau selbst greift. Er erweitert seine Betrachtungen zur Geschichtsschreibung als Bestattungsritus durch begriffliche Drehungen, die nicht umsonst den Begriff des tropus in den Mittelpunkt seiner Kunst des Handelns stellt. Dies geschieht nicht konstativ, sondern als Theorieperformanz, die witzig genug, von Freud geradewegs zu Clausewitz überspringt. ${ }^{27}$ Denn es sind Ta-

23 Sigmund Freud: Das Unbehagen in der Kultur (1930). Frankfurt a. M. 1974, S. 74.

24 Jean Paul: Vorschule der Ästhetik. Nach der Ausgabe von Norbert Miller hg. von Wolfhart Henckmann. Hamburg 1990, S. 129.

25 Stephen Greenblatt: Shakespearian Negotiations. The Circulation of Social Energy in Renaissance England. Berkeley 1988, bes. S. 1. Vgl. Jürgen Pieters: Moments of Negotiation. The New Historicism of Stephen Greenblatt. Amsterdam 2001, bes. S. 124-139.

26 Certeau: Mystische Fabel (wie Anm. 20), S. 130f.

27 Dass Certeau (Kunst des Handelns [wie Anm. 17], S. 90) selbst zu einer List, nämlich einem korrumpierten Zitat greift, zeigt sich, wenn man auf die Quelle Clausewitz zurückgeht. Clausewitz schreibt der List nicht den Wert zu, den Certeau durch Clausewitz reklamiert. Clausewitz fährt nämlich nach der von Certeau zitierten Stelle so fort: „Aber so sehr man gewissermaßen das Bedürfnis fühlt, die Handelnden im Krieg an verschlagener Tätigkeit, Gewandtheit und List sich einander überbieten zu sehen, so muss man doch gestehen, dass diese Eigenschaften sich in der Geschichte weig zeigen und selten aus der Masse der Ver- 
schenspielereien mit Ideen und Vorstellungen wie mit Handlungen, die Möglichkeiten von Veränderungen bedingen und damit Zukunft sichern:

Mit Hilfe der Vorgänge, die Freud am Beispiel des Witzes darstellt, werden dabei [bei Clausewitz] frech Elemente miteinander in Verbindung gebracht, die etwas anderes in der Sprache eines Ortes aufblitzen lassen und den Adressaten verblüffen sollen. Streiflichter, Knalleffekte, Risse und Volltreffer in den Netzen eines Systems. ${ }^{28}$

\section{Philosophiehistorische Verortung und philologische ,Stellen`}

(Descartes vs Bacon; Johann Georg Hamann und Jean Paul)

Um solche „Volltreffer in den Netzen eines Systems“ geht es im Folgenden. Hamann und Jean Paul werden nicht literaturgeschichtlich eingesargt, ihre „Knalleffekte“ sollten die noch Lebenden aus dem Dämmerschlaf der Vernunft wecken: In der Kulturgeschichte gibt es Ereignisse, die noch gar nicht zur Kenntnis genommen wurden, obwohl sie uns selbst bestimmen. Um die Einsicht in den eigenen kulturellen Quellcode zu bekommen ist eine rhetorikgeschichtliche Modifikation des von Certeau gegebenen Hinweises auf Clausewitz' Relation von Trope und Krieg hilfreich, ${ }^{29}$ die zugleich eine wesentliche Vorbedingung für Hamann wie Jean Paul aufspürt, die sich gerade dort zeigt, wo die Rhetorik scheinbar schon verschwunden ist, nämlich im Cartesischen Programm.

Descartes, dessen Philosophie auch als Auseinandersetzung mit dem Dreissigjährigen Krieg zu verstehen lohnt, leitete in den Regeln zur Ausrichtung der Erkenntniskraft (1628) und Die Leidenschaften der Seele (1649) das menschliche Redevermögen nicht nur allein aus natürlichen Gegebenheiten ab, sondern führte es auf physikalische Gründe zurück. Er schied also die Rhetorik nicht nur als Erkenntnisgenerator aus, sondern rehabilitierte sie implizit als Theorie sprachgebundenen Denkens. Die Erkenntnis dieser Komplexität drang jedoch in Deutschland erst Mitte des 18. Jahrhunderts durch, während die katholischen Intellektuellen Frankreichs in der Logik von Port Royal, etwa in Antoine Arnaulds Die Logik oder die Kunst des Denkens (1662) schon sehr früh die carte-

hältnisse und Umstände sich haben hervorarbeiten können." Carl von Clausewitz: Vom Kriege. Vollständige Ausgabe. Hamburg 2014, S. 209.

28 Certeau: Kunst des Handelns (wie Anm. 17), S. 90.

29 Deutlicher als die von Certeau zitierte Stelle scheint folgende Bemerkung von Clausewitz zu sein: „[...] der Bedrängte wird, kaum Hilfe mehr erwartend von Dingen, die ihm keine versprechen, sein ganzes und letztes Vertrauen in die moralische Überlegenheit setzen, welche die Verzweiflung jedem Mutigen gibt, er wird die höchste Kühnheit als die höchste Weisheit betrachten, allenfalls noch kecker List die Hand reichen und, wenn kein Erfolg ihm werden soll, in einem ehrenvollen Untergang das Recht zu künftiger Auferstehung [!] finden." Clausewitz: Vom Kriege (wie Anm. 27), S. 310. 
sische Erkenntniskritik zum Zwecke der Verteidigung der Erbsündenlehre radikalisierten. In diesem Kontext versuchte Bernard Lamy den philosophischen Aktivitäten in Descartes' Untersuchung der Leidenschaften bis in ihre feinsten rhetorischen Verästelungen zu folgen, kam aber mit denselben analytischen Prozeduren zu ganz anderen Ergebnissen als Descartes und Arnauld. Seine Kunst zu reden analysierte zwar die menschliche Seele ebenfalls unter dem Aspekt der physis. Die Figuren der Rhetorik sind demnach schlicht durch die Leidenschaften des Menschen erklärbar, die wie die logischen Kräfte auch zu seiner Natur gehören. Die Rede folgt also mithilfe der rhetorischen Figuren lediglich seelischen Bewegungen. Wenn etwa die Seele von einer gewaltsamen Bewegung beunruhigt wurde, so laufen die cartesianischen Lebensgeister durch alle Teile des Leibes, lassen die Muskeln kontrahieren und so auch die Stimme erhöhen und nachdrücklich werden. Alle Figuren können so natürlich erklärt werden. So wie jeder Fechter in seiner Todesnot ohne jede Kunst gerade zu den Finten und Stellungen greift, die ihn retten, ${ }^{30}$ so stellt uns auch die Natur die Figuren als Finten zur Verfügung.

Dieses allzu dichotomische Schema von cartesischer Rhetorikkritik und Lamys Konzept einer natürlich begründeten Rhetorik überzeugte zwar gerade in Deutschland einen grossen Kreis von Aufklärern (Wolff, Gottsched). Aber das Problem, dass sich Lamy zwar vom engmaschigen Systembegriff des cartesischen Ego löst, ohne ihm jedoch entkommen zu können, wurde hier nicht weiter reflektiert, sondern unter dem Namen einer, vernünftigen Rhetorik' nur bestätigt. Trotz seiner Fintenmetaphorik gelang es Lamy nicht, die cartesische Logik selbst in Frage zu stellen, sondern nur die Rhetorik im Namen der Leidenschaft anthropologisch zu rechtfertigen. Dass aber der Status des Individuums im Masse der logizistischen Expansion schwand, liess sich dadurch nicht mehr bremsen. Die Überlistung des Systemdenkens wurde erst durch Hamanns philologische Experimente im Rückgang auf Francis Bacon möglich: „Denn wie die experimentierenden Naturforscher einen Körper in allerhand willkührliche Verbindungen mit andern Körpern versetzen und künstliche Erfahrungen erfinden, seine Eigenschaften auszuholen ${ }^{\text {“31 }}$ so soll es laut Hamann jegliche Philologie mit ihren Texten machen.

30 Bernard Lamy: De l'art de parler. Kunst zu reden. Hg. von Ernstpeter Ruhe. München 1980, S. 122-126.

31 „Ein sorgfältiger Ausleger muß die Naturforscher nachahmen. Wie diese einen Körper in allerhand willkührliche Verbindungen mit andern Körpern versetzen und künstliche Erfahrungen erfinden, seine Eigenschaften auszuholen; so macht es jener mit seinem Texte. Ich habe des Sokrates Sprüchwort mit der Delphischen Überschrift zusammen gehalten; jetzt will ich einige andere Versuche thun, die Energie desselben sinnlicher zu machen." N II, $71,25-31$. 
Die philologischen Experimente sollen bei Hamann diktatorische Geltungsansprüche sei's der Vernunft, sei's der Offenbarungstheologie aufbrechen. Der Philologe soll „in kleinen Heften mit Zweifeln und Einfällen gegen die Dictatoren der reinen Lehre und Vernunft [...] laboriern. ${ }^{\text {32 }}$ Hamanns scharfsinniges philologisches Laborieren als Spiel mit den Ordnungen der reinen Lehre und Vernunft erweist sich gerade im Kontext von Natur und Geschichte als Adaption Francis Bacons im Kontrast zur cartesischen Philosophie. Bacon beschränkte Experimente nicht nur auf die Natur. Seine Temporis partus masculus (1603) verwendet Zitate und Bilder, um wie Platon in seiner Politeia den Lesenden gerade auf seine Gefangenschaft in Zitaten und Bildern aufmerksam zu machen.

Wesentlich für Hamann wie Jean Paul ist, dass Bacon mit den Lesenden ein Experiment unternimmt. Er lässt in seinen Texten verschiedene Bildbegriffe als Bilder von Bildern wie in einem Film vor ihnen ablaufen. Sein Text dynamisiert damit den Bildbegriff selbst: Zunächst sind die Idole statische Bilder, dann aber jene dynamischen Visionsexperimente, wie sie die Alchimisten seiner Zeit unternahmen, wenn sie heterogene Bildfelder miteinander kombinierten, um zu neuen Erkenntnissen vorzustossen.

Schliesslich aber geht Bacon über die Metaphorik des Bildes als Schatten zum Unbildlichen, ja Unbegrifflichen über. Sein ikonoklastischer Text verweigert sich wie die spätere moderne Literatur hermeneutischer Entschlüsselung: Denn eben sie ist durch jene Bilder bestimmt, die uns lenken, und die es gerade hinter sich zu lassen gilt. Die Philosophie kann zwar den Geist von den Idolen befreien, indem sie diese argumentativ zerstört. Im Unterschied zu Descartes ist sich Bacon aber darüber im Klaren, dass die angestrebte Produktion neuen, sicheren Wissens nicht durch die Angabe isolierter Daten gelingt, sondern nur dort, wo Wissen in einem in sich begründeten Zusammenhang steht, der auf die Selbstvermehrung des Wissens zielt. Für diesen Zusammenhang verwendet Bacon in Valerius Terminus (1603) das Bild von den Wissenschaften als eines Kreises, ,Enzyklopädie‘, den es zu durchlaufen gilt. Invariante dieses Durchlaufs sind ,Aphorismen', jene transdisziplinären Axiome, die von mehreren wissenschaftlichen Disziplinen gemeinsam geteilt werden, scheinbar blosse Leerformeln, die mit den empirischen Beobachtungen der Einzelwissenschaften jeweils zu füllen sind. Der gesuchte Zusammenhang des Wissens lässt sich darum nicht mit den wissenschaftlichen Mitteln selbst erzeugen. Diese sind nur Elemente einer Komposition, einer Wissenschaftsästhetik, die durch das Prinzip des Aphorismus organisiert ist. ${ }^{33}$

32 N IV, 460.

33 Christian Sinn: ,[...] diese Wissenschaft ist noch nicht vorhanden.` Der wissenschaftsästhetische Entwurf einer Allgemeinen Methodenlehre an der Wende zum 19. Jahrhundert als Grundlage romantischer Textproduktion. In: Internationales Jahrbuch der Bettina-vonArnim-Gesellschaft 16 (2004), S. 27-56. 
Der Aphorismus dient bei Bacon als Mittel zur Erreichung staatspragmatischer Räson: er will dem heuristischen Prinzip aller Wissenschaften auf die Spur kommen, so dass durch methodisch kontrollierte wissenschaftliche Interdependenz neue, für den Menschen nützliche Entdeckungen und Erfindungen möglich werden. Hierzu aber bedarf es der Phantasie: Sie abduziert die Aphorismen. In De augmentis (1623) fordert Bacon deshalb nicht nur Phantasie, er aktiviert sie selbst in anderen Texten, die mit ihrer Rhetorik gezielter Unbestimmtheit uns heute noch vor den Kopf stossen und geradezu ,dekonstruktivistisch` anmuten.

Sie entstammen jedoch einer alten Technik der philosophischen Rhetorik, die ein Training im Denken durch Prolepsen, d.h. durch Vorausnahmen und Vorausdeutungen, herzustellen versuchte. Durch diese rhetorische Figur werden allgemein Zeichen in ihnen fremde Kontexte gestellt, so dass neue Entdeckungsmöglichkeiten hergestellt werden. Bei Bacon geschieht dies, wie dann bei seinen späteren Bewunderern Johann Georg Hamann und Jean Paul, durch die Verwendung korrupter Zitate, die den Lesenden auffordern, die Bedingungen seiner Rezeption zu problematisieren. Denn Bacons Annahme, dass unser Bewusstsein von Natur aus falsch und fälschend sei, hat zur Folge, dass eine Therapie nicht durch die Natur, sondern allenfalls durch literarische Konstruktionen gelingt. Aber auch wenn nicht Natur, sondern Tradition als Ursache angenommen wird, kann Literatur durch die bewusste Korruption von Zitaten solche Tradition vernichten.

Literatur wird unter diesem therapeutischen Aspekt mit Aufklärung synonym: Denn einerseits genügt es nicht, in den Spiegel des eigenen Bewusstseins zu schauen, um die Realität zu erkennen, da dieser Spiegel, sei es durch die Natur selbst, sei es durch die Tradition, verzerrt ist. Weil Realität jedoch nur durch diesen Spiegel wahrnehmbar ist, bleibt allenfalls der literarische Umgang mit den Bedingungen der Möglichkeit von Konstruktionen übrig, um sich über sich selbst aufzuklären: Darin besteht die Aufklärungsleistung der Literatur, die Hamann und Jean Paul gleichermassen interessiert. Sie stellen, wie dies Walter Benjamin in expliziten Anschluss an Hamanns auch tut, ${ }^{34}$ die Vernunft in den Zwischenraum der durch den Sündenfall bereits gebrochenen paradiesischen Sprache, die aber noch nicht durch Babel hindurch gegangen ist.

Denn Vernunft, exemplarisch fassbar im cartesischen Programm, insinuiert zwar nicht paradiesische, aber vorbabylonische, d.h. historisch gesehen un-

34 Walter Benjamin: Über das Programm der kommenden Philosophie (1917). In: Gesammelte Schriften, unter Mitwirkung von Theodor W. Adorno und Gershom Scholem hg. von Rolf Tiedemann und Hermann Schweppenhäuser. Bd. II. Frankfurt a. M. 1977, S. 168: „Die grosse Umbildung und Korrektur die an dem einseitig mathematisch-mechanischen orientierten Erkenntnisbegriff vorzunehmen ist, kann nur durch eine Beziehung der Erkenntnis auf die Sprache wie sie schon zu Kants Lebzeiten Hamann versucht hat gewonnen werden." 
haltbare Ansprüche, die das nachbabylonische Faktum der Zerstreuung nicht zu akzeptieren bereit ist, so dann auch die spätere Argumentation Derridas im Anschluss an Benjamin. Wenn es aber nach Babel weder einen Urtext noch eine Ursprache mehr gibt, so folgt daraus, dass sich die Sprachen nicht so ineinander übersetzen lassen, dass sich daraus Machtansprüche der Vernunft und seien es die einer gewaltfreien Kommunikationsgemeinschaft a la Habermas ableiten lassen. Denn Gott selbst verhindert radikaltheologisch gesprochen durch die Dekonstruktion seiner selbst die Machtergreifung der menschlichen Vernunft.

Die Dekonstruktion Gottes durch Gott selbst ist der Grund für eine bewusst hergestellte petitio principii bei Hamann und in anderer Weise aber auch bei Jean Paul: Natur und Geschichte sollen den Zugang zum göttlichen Wort erst ermöglichen, dieses Wort ist jedoch bereits die Voraussetzung dafür, Natur und Geschichte zu verstehen. Was für den analytischen Philosophen nach definitionslogischem Unfug klingen muss, wird von beiden Autoren jedoch dazu verwendet, das Denken durch eine Aporie auf den Grund und die Grenze seiner selbst aufmerksam zu machen, die sich durch den Ausdruck, Gott' nur als unbeholfenes Kürzel eines reflektierten Agnostizismus benennen lässt.

Dieses von Hamann und Jean Paul gemeinsam geteilte Problem, ob das Denken selbst die durch es erzeugten Differenzen vermitteln kann, lässt sich zum einen an der von beiden Autoren geteilte These belegen, dass es ein commercium mentis et corporis gebe, das das spekulative Erkenntnisvermögen der Vernunft überfordere: Wie können denn Logik und Leidenschaft in ein und demselben Individuum existieren? Diese Frage hatte Descartes offen gelassen und einseitig logizistisch beantwortet; Lamy hatte lediglich die Leidenschaften und mit ihnen die Rhetorik rehabilitiert. Hamann und Jean Paul argumentieren umgekehrt: Das commercium existiert ja bereits in unserer Verbindung von Leib und Seele, kann aber durch Vernunft und Logik nicht aufgelöst werden. ${ }^{35}$ Diese Überforderung der Vernunft ist eine Art negativer Gottesbeweis dafür, dass die Menschen bereits selbst ein Kommentar zum Wort Gottes sind wie dies auch die Konjunktion von Natur und Geschichte ist.

Obwohl Hamann und Jean Paul die Metapher von den beiden Büchern der Natur und der Geschichte häufig verwenden, treten zwischen ihnen deutliche Unterschiede auf. Zunächst Hamann: „Alle Erscheinungen der Natur sind Träume, Geschichte, Rätsel, die ihre Bedeutung, ihren geheimen Sinn haben. Das Buch der Natur und der Geschichte sind nichts als Chiffern, verborgene Zeichen,

35 Vgl. Jean Pauls sämtliche Werke. Historisch-kritische Ausgabe. Im Auftrag der Preussischen Akademie der Wissenschaften begr. von Eduard Berend. Weimar 1927-. Im Folgenden zitiert als ,HKA'. Hier: HKA, Abt. II, Bd. 1, S. 283: „Die Vereinigung unseres Körpers mit unserer Seele bleibt das ewige Rätsel jedes Philosophen; wir wissen nicht, sol er [!] unsre Weisheit oder Torheit, unser Glük oder Unglük befördern; uns ist unbekannt, was wir ihm zu danken haben, wenig, viel oder alles.“ 
die eben den Schlüssel haben, der die heilige Schrift auslegt. “36 Mit diesem Schlüssel meint Hamann den Glauben des vom Heiligen Geist erfüllten Menschen. Er allein erfasst den Sinn der Bibel, er allein erfasst den Sinn der Natur und der Geschichte: „Natur und Geschichte [...] sind die zwei grossen Commentarii des göttlichen Wortes, und dieses hingegen der einzige Schlüssel, uns eine Er-

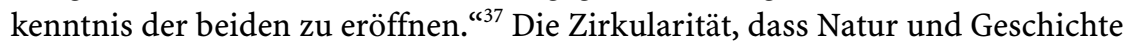
das Wort Gottes veranschaulichen und erklären und der Schlüssel zu diesem Wort sind, obwohl erst dieses Wort die Chiffernschrift von Natur und Geschichte zu lesen vermag, löst Hamann zwar durch einen Sprung in den Glauben auf. Das ist in wissenschaftlicher Hinsicht zunächst wenig ergiebig. Der Glaube ist für Hamann das Aufnahmeorgan für die göttliche Offenbarung, Offenbarung ist ihm aber identisch mit Erfahrung. ${ }^{38}$ Glaube bedeutet bei Hamann das Aufnehmen sinnlicher und übersinnlicher Objekte in einer bestimmten Perspektive, die dem Kult der Vernunft eine im menschlichen Geist selbst verankerte Religion entgegensetzt. Diese natürliche Religion approximiert Hamann durch das interkulturell nachweisbare poetische Vermögen des Menschen. Poetisierung der Religion ist daher bei ihm keine nachträgliche rhetorische Einkleidung von Glaubenssätzen, sondern Begründung von Religion. ${ }^{39}$ Die christliche Religion

$36 \mathrm{~N} \mathrm{I}, 308,32-36$.

$37 \mathrm{~N} \mathrm{I}, 35-37$.

38 N I, 70,37-40: „Alles ist Weisheit in Deiner Ordnung der Natur, wenn der Geist Deines Wortes den unsrigen aufschliesst. Alles ist Labyrinth, alles Unordnung, wenn wir selbst sehen wollen.“; N I, 76,2-6: „Wollen wir etwas wissen, so lasset uns den Geist fragen, der über der Tiefe schwebt, der diese ungestalte, leere, geheimnisvolle Welt in die Schönheit, die Klarheit, die Herrlichkeit versetzen kann, gegen welche die übrige Schöpfung ihren Glanz zu verlieren scheint.“; N I, 128,7-14: „Wer den Geist Gottes in sich fühlt, der wird ihn gewiss auch in der Schrift fühlen [...] So wahr ist es, dass seine Absicht gewesen, keinen andern als den Gläubigen zu gefallen. Der Ungläubige geht ihn nichs an [...] Der Gläubige ist allein sein Vertrauter [...]“; N I, 246,26-27: „Ohne Glauben können wir selbst die Schöpfung und die Natur nicht verstehen “; N III, 191,27-34: „Weil unsere Vernunft blos aus den äußeren Verhältnissen sichtbarer, sinnlicher, unstätiger Dinge den Stoff ihrer Begriffe schöpft, um selbige nach der Form ihrer innern Natur selbst zu bilden, und zu ihrem Genuß und Gebrauch anzuwenden: so liegt der Grund der Religion in unserer ganzen Existenz und außer der Sphäre unserer Erkenntniskräfte, welche alle zusammengenommen, den zufälligsten uns abstractesten modum unserer Existenz ausmachen.“; N III, 190,16-23: „Da der Glaube zu den natürlichen Bedingungen unserer Erkenntniskräfte und zu den Grundtrieben unserer Seele gehört; jeder allgemeine Satz auf guten Glauben beruht, und alle Abstractionen willkührlich sind und seyn müssen: so berauben sich die berühmtesten Speculanten unserer Zeit über die Religion ihrer Vordersätze und Mittelbegriffe, die zur Erzeugung vernünftiger Schlußfolgen unentbehrlich sind [...].“; 22.4.1787 an Jacobi, ZH VII, 156,20-24: „Ich will aber den berlinischen Idealismus des Christentums und Luthertums widerlegen durch einen historischen u physischen Realismum. Erfahrung der reinen Vernunft entgegensetzen. Diese Verwickelungen ins Reine zu bringen, ist eben die herkulische Arbeit, die mir im Sinne liegt [...].“

39 N III, 191,34-192,4: „Daher jene mythische und poetische Ader aller Religionen und ihre Torheit und ärgerliche Gestalt in den Augen einer heterogenen, inkompetenten, eiskalten, 
steht mit ihrem Ärgernis des Kreuzes nur stellvertretend für das Ärgernis, das alle Religionen für die Vernunft erzeugen. ${ }^{40}$ Der Rationalismus ist nur eine andere Form des Despotismus, gegenüber dem das Werk der Reformation noch gar nicht in Gang gesetzt wurde. ${ }^{41}$ Vor allem aber verkennt die Aufklärungsreligion das eigentümliche Wesen von Religion, wenn sie Gott zu beweisen versucht. ${ }^{42}$ Von dieser Auffassung aus ist einerseits verständlich, dass sich Hamann manchmal dem Immanenzgedanken des Pantheismus annähern kann, ${ }^{43}$ ihn hiervon jedoch die geschichtliche Begründung des christlichen Erlösungsglaubens trennt. ${ }^{44}$

Bei Jean Paul liegen die Dinge auf den ersten Blick ganz anders als bei Hamann. Mystische Auslegungen des Bibeltextes lehnt Jean Paul bewusst und entschieden ab, er lässt nur eine gelten, die historisch-kritische im Anschluss an Johann Salomon Semmler in Halle. Jean Paul steht zwar im engsten Kontakt mit Hamann, Herder und Jacobi. Als aber um die Jahrhundertwende und mit den Befreiungskriegen als Rückschlag gegen denn Rationalismus die romantischmystische Richtung des Christentums immer mehr Boden gewann und auch ihm nahestehende Personen wie Jung-Stilling, Krüdener, Kanne, vor allem aber sein eigener Sohn Max sich dieser Bewegung anschlossen, schwieg Jean Paul nicht länger, sondern bekannte offen seinen Standpunkt. Wesentlich ist hier die Streitschrift „Ueberchristentum. Wider Kanne“. Zunächst stellt Jean Paul hier den Grundsatz auf, dass älter und wichtiger als die Bibel ein bestimmter Begriff von Christentum sei. Die Bibel ist nur die, wenn nicht sogar nur eine Urkunde dieses Christentums. ${ }^{45}$ Aus diesem Grund, der sich einfacher als praktische

hundemagren Philosophie, die ihrer Erziehungskunst die höhere Bestimmung unserer Herrschaft über die Erde unverschämt andichtet."

40 N III, 225,3-6: „Was ist die hochgelobte Vernunft mit ihrer Allgemeinheit, Unfehlbarkeit, Überschwenglichkeit, Gewissheit und Evidenz? Ein Ens rationis, ein Ölgötze, dem ein schreyender Aberglaube der Unvernunft göttliche Attribute andichtet"

41 An Jacobi 22.7.1785, ZH VI, 22,31-34: „Anstatt des römischen [Despotismus] ist ein metaphysisch-moralischer in der Mache, der seinen Sitz an eben dem Orte hat, wo man soviel Zetergeschrei über das seel. Pabst[th]um erhebt."

42 An Jacobi 18.2. 1786, ZH VI, 277,24-26: „[W]enn die Narren sind, die in ihrem Herzen das Daseyn Gottes leugnen; so kommen mir die noch unsinniger vor, die selbiges erst beweisen wollen“. N III, 319,27-29: „Das Daseyn Gottes leugnen und beweisen wollen, ist im Grunde, wie der seelige Voltaire [!] sagt: Sottise de deux parts!“

43 N III, 165,15-19: „ob nicht die Perle des Christentums ein verborgenes Leben in Gott, eine Wahrheit in Christo, dem Mittler und eine Kraft sein müsse, die weder in Worten und Gebräuchen noch in Dogmen und sichtbaren Werken besteht, folglich auch nicht nach dialectischen und ethischen Augenmaaße geschätzt werden kann“.

44 An Jacobi 5. 12.1784, ZH V, 274,22 f.: „Läst sich wol mit dem panischen System im Kopf ein christlich Vaterunser beten?"

45 Jean Paul: HKA (wie Anm. 35), Abt. II, Bd. 4, S. 62 (340): „Befestigt doch nicht die Bibel, deren Leben an der historischen Zeit anhangt, sondern lieber ihre Grundsätze, d.h. die Grundsätze, auf denen ihr Heiliges allein ruht und die vorangehen und die sie voraussetzten 
Nächstenliebe übersetzen liesse, hält Jean Paul nicht sehr viel von den Inhalten des Alten Testamentes. ${ }^{46}$

Während für Hamann die menschliche Sprache notwendig metaphorisch ist, weil sie dem von Gott geschriebenen Buch der Natur entstammt, neigt Jean Paul zur umgekehrten Argumentation. Da bei ihm die menschliche Sprache durch Metaphern den Konnex zwischen Quantitäten und Qualitäten stiftet, kann sie als Indiz für einen göttlich begründeten Zusammenhang verstanden werden, in der die Welt selbst zum Zeichen wird. Schematisch gesprochen verschiebt sich im Übergang von Hamann zu Jean Paul der Akzent vom Finden, auch sich Findenlassen des Menschen durch Gott zu einem Erfinden, in dem das Buch der Natur zum Palimpsest des Witzes wird. Jean Paul bezeichnet den erfinderischen Witz als Anagramm der Natur und als „Geister- und Götterleugner“. ${ }^{47}$

In diesem Kontext Jean Pauls dient nun nicht wie bei Hamann Rhetorik dazu, Theologie zu ermöglichen, vielmehr werden Theologumena zu Elementen des Witzes $^{48}$, so dass die Welt zu einem völligen Chaos wird, über welchem ein „heiliger Geist“ schwebt; sie muss ihren „Jüngsten Tag“ erleben, damit der Geist des poetischen Ich ganz frei und autonom sein soll. Nur die durch den Witz hergestellte „allgemeine Gleichheit und Freiheit“ eröffnet den „Weg zur dichterischen und zur philosophischen Erfindung". ${ }^{49}$ Beachtete man nicht die weiteren Kontexte der Vorschule der Ästhetik, aus der diese Paraphrase stammt, so könnte man nach der soeben skizzierten positivistischen Gegenüberstellung von Zitatcollagen einen Gegensatz zwischen Hamann und Jean Paul vermuten. Aber

muss. Tut nicht als ob ohne die Bibel das Höchste verloren sei, sondern erkennt das Höchste für sich und dann könnt ihr etwa wieder Bibeln darauf gründen"

46 Ebd., S. 48 (61): „Ich möchte wissen, was ein Unbefangener, dem kein neues Testament eine höhere Beziehung vom alten beigebracht hätte, von diesem dächte, von dessen sämtlichen unsittlichen Helden, von den Mordgrundsätzen. In allen Evangelien und Worten Christi finde ich nichts, was der Vernunft widersteht; erst später hinter den Aposteln kam es zu Erweiterungen und Widersprechungen.“; ebd., S. 43 (36): „Ich leugne nichts, was in der Bibel steht, aber das Meiste, was die Leute hineintragen. Die Bibel, d.h. das neue Testament [!], verträgt sich mit jeder Philosophie, die an Gott glaubt.“

47 Jean Paul: HKA (wie Anm. 35), Abt. I, Bd. 5, S. 201: „Der Witz - das Anagramm der Natur ist von Natur ein Geister- und Götter-Leugner, er nimmt an keinem Wesen Anteil, sondern nur an dessen Verhältnissen; er achtet und verachtet nichts; alles ist ihm gleich, sobald es gleich und ähnlich wird; [...] er ist atomistisch, ohne wahre Verbindung.“

48 Ebd., S. 202: „Nun gibt es einen lyrisch-witzigen Zustand, welcher nur aushungert und verödet, wenn er bleibt und herrscht, aber wie das viertägige Fieber die herrlichste Gesundheit nachlässet, wenn er geht. Wenn nämlich der Geist sich ganz frei gemacht hat [...], wenn zwar ein Chaos da ist, aber darüber ein heiliger Geist, welcher schwebt, [...] wenn dieser Dithyrambus des Witzes [...] den Menschen mehr mit Licht als mit Gestalten füllt: dann ist ihm durch die allgemeine Gleichheit und Freiheit der Weg zur dichterischen und zur philosophischen Freiheit und Erfindung aufgetan, und seine Findkunst (Heuristik) wird jetzo nur durch ein schöneres Ziel bestimmt."

49 Ebd. 
auch wenn diese philologischen ,Belege` zuvor eher als Travestie der Philologie missbraucht wurden, so bringen sie trotzdem schon das zum Vorschein, was die Zitate über sich „zur philosophischen Erfindung“ im Geiste Bacons hinaustreibt. Doch mag, um der wissenschaftlichen Praxis Genüge zu tun, die philologische Spurensuche noch ein wenig weiter verfolgt werden.

Während Hamann das Buch der Natur als Chiffernschrift des biblischen Gottes versteht, bleibt Jean Paul in der Bestimmung der Autorschaft dieses Buches unklar. Einerseits sieht er in der Erscheinungswelt der Natur ein eher allgemein göttliches Wesen, andererseits aber ist es das Genie, dem zugesprochen wird, die Natur als Buch wortwörtlich zu buchstabieren. Gegenüber Hamann scheint dies zunächst eine eher Kantianische Position zu sein, in der der Mensch die Lesart dieses Buches bestimmt, ja es allererst schreibt. Jedoch unterscheidet Jean Paul von Kant der Blick auf jenes Buch, das ihm wichtiger als das der Natur ist, nämlich das Buch der Geschichte, das ihn geradewegs zu Hamann zurückführt.

Zwar gilt auch hier für Jean Paul, allerdings mehr von Vico als von Kant ausgehend, dass die Menschen das Buch der Geschichte selbst gemacht haben. Aber, und das ist das zentrale, mit Hamann geteilte Argument, die Geschichte und vor allem die Geschichtlichkeit der Sprachen ist konstitutiv für die menschliche Vernunft. Dies ist bereits für den jungen Jean Paul entscheidend. In einer Denkübung von $1780^{50}$ geht es ihm um die Bestimmung aller Geschöpfe zur fortschreitenden Vervollkommnung. Jede Gattung strebt hier einem Idealzustand entgegen und jedes Tier entwickelt sich im Lauf seines Lebens so weit, dass es die Grenzen der in seiner Gattung selbst angelegten Möglichkeiten erreicht, es repräsentiert also diese Gattung erschöpfend. Anders der Mensch: nur als geschichtliches Ganzes nähert sich sein Geschlecht dem Endpunkt möglicher Vervollkommnung, der Einzelne aber ist blosses Glied einer Kette.

Während sich die Tierindividuen nicht beliebig miteinander verständigen können, gelingt dies den Menschen qua ihrer geschichtlich gewordenen Sprache verständigen. So entwickelt sich die Menschheit über jeden tierischen Vervollkommnungsgrad hinaus bis zur „Stufe des Engels“. ${ }^{51}$ Sprachgeschichte wird für den jungen Jean Paul zwar noch nicht als Vernunft und Kritik der Vernunft formuliert, jedoch das Buch der Geschichte als gattungskonstitutiv anerkannt.

Trotz ihrer erheblichen Differenzen teilen daher Hamann und Jean Paul das Problem, dass Natur und vor allem Geschichte als Kommentare des göttlichen Wortes nötig wären, um das göttliche Wort überhaupt verstehen zu können, das

50 Jean Paul: HKA (wie Anm. 35), Abt. II, Bd. 1, S. 42 (32): „Alles ist Sele - aber eine Sel' ist nur besser als die andre. Vom Menschen bis zum unförmlichen Kiesel herrscht Vervollkommnung Seiner Selbst. [...]“

51 Ebd. S. 49. 
Verständnis dieses Wortes aber die Voraussetzung ist, Natur und Geschichte als Kommentare zu verstehen. Der Schlüssel des göttlichen Wortes machte die Allegorien der Natur und der Geschichte erst lesbar. Wer wagte es aber, solche Schlüsselgewalt für sich zu beanspruchen? Allenfalls wären Konjekturen möglich, wie sie Jean Paul dem Genie zuspricht - ethisch vielversprechender scheint jedoch jener allgemeine Verzicht auf Auslegungshoheit zu sein, den Hamanns philologische Experimente intendieren.

Zwar legt Hamann in einer gut lutherischen subtilitas applicandi eine partiale Auflösung des definitorischen Problems durch Anwendung auf die eigene Lebenssituation nahe. ${ }^{52}$ Aber auch solche Lebenszeugnisse sind wie die zwei grossen ,Commentarii' durch die Medien korrumpierter Schrift und Rezeption gegangen und wurden oftmals durch rhetorische Mittel allererst hergestellt und ,optimiert'. Hamann und Jean Paul wissen das als Rhetoriker natürlich, während die theologischen, philosophischen, soziologischen Lesarten von Hamann und Jean Paul diesen letztlich philologischen Sachverhalt geflissentlich übersehen.

Das Problem der zirkulären Definition des Kommentars des göttlichen Wortes durch Natur und Geschichte bleibt indes ebenso wenig auf sich beschränkt wie die dunklen Beziehungen zwischen körperlicher und seelischer Natur, zwischen individueller und sozialer Stilistik und zwischen sprachlichen und vorsprachlichen Bedingungen des Denkens. Diese vier gemeinsamen Elemente im Denken von Hamann und Jean Paul sind nur ein Ausdruck für das allgemeine principium coincidentiae oppositorum, nach dem beide Autoren suchen. ${ }^{53}$

Hamann und Jean Paul versuchen in je unterschiedlicher Weise die durch das Denken hergestellten Differenzen durch eine sprachgeschichtliche Selbstreflexion aufzulösen. Entscheidend hierfür ist, dass das im Übergang von Cusanus zu Bruno formulierte principium coincidentiae oppositorum in der zweiten Hälfte des 18. Jahrhunderts im Kontext einer transzendental-hermeneutischen Sprachreflexion durch Hamann und Herder neu gedacht wird. Alle bisher genannten Oppositionen, z.B. die von Körper und Geist, Individuum und Gesellschaft,

52 N I, 297,25-30: „Ich bin überzeugt, daß jede Seele eine Schaubühne so grosser Wunder ist, als die Geschichte der Schöpfung und der ganzen heiligen Schrift in sich schlüst. Der Lebenslauf jedes Christen ist im Tagewerke Gottes, in Bündnisse desselben, mit dem Menschen, in Uebertretung, Warnung, Offenbarung, wunderthätigen Erhaltung pp. begriffen“.

53 An Herder 29.4.1781, ZH IV, 287: „Nichts scheint leichter als der Sprung von einem Extrem zum andern und nichts ist so schwer, als ihre Vereinigung zu einem Mittel. Ungeachtet aller meiner Nachfrage ist es mir nicht möglich gewesen, des Jordanus Brunus Buch de Uno aufzutreiben, worin er sein principium coincidentiae erklärt, das mir Jahre lang im Sinne liegt, ohne dass ich es weder vergessen noch verstehen kann [...] Diese Coincidenz scheint mir der einzig zureichende Grund aller Widersprüche und der wahre Process ihrer Auflösung und Schlichtung, aller Fehde der gesunden Vernunft und reinen Unvernunft ein Ende zu machen.“; vgl. Jean Paul: Vorschule der Ästhetik (wie Anm. 24), $\$ 43$. 
Sprache und Nichtsprache, und eben auch von Natur und Geschichte gründen in der Tiefenopposition von Zeichen und Bezeichnetem. Zeichen werden nicht mehr wie zuvor einem Bewusstseinsinhalt nachträglich zugeordnet, vielmehr wird, bereits einer dreistelligen Semiotik nach dem Muster von Peirce präludierend, die unendliche semiosis und die konstitutive Bedeutung des Sprechens für das Denken differenziert anerkannt. Interessant ist jedoch, dass das Sprechen und die Sprache nicht einseitig als transzendentaler Grund des Denkens formuliert werden, vielmehr rückt damit, deutlicher bei Hamann und dann Jean Paul als bei Herder die Verführung des Denkens durch die Sprache erst recht in den Blick.

\section{Surge amica mea: Der Vogel Merops}

(Pascal, Luther, Hamann, Jean Paul)

Soll nach dem Vorschlag Hamanns der Theologe mit der Geschichte als einem Kommentar des Wortes Gottes wie ein Naturwissenschaftler nach dem Vorbild Bacons vorgehen, dann ist damit auch zugleich die mögliche Kongruenz von Natur und Geschichte gegeben. Von Bacon ausgehend lässt sich eine poetologische Linie zu Hamann und Jean Paul ziehen (wie ich in meiner Dissertation belegt habe).$^{54}$ Innerhalb dieser Linie sind Zitate bei Hamann und Jean Paul dann eben nicht ,Belegstellen' für Theologoumena und Philosopheme, die sich durch Theologie und Philosophie verwalten liessen, sondern Poetisierungen des Wortes Gottes durch eine sich selbst negierende Intertextualität. Ihre konstative Differenz, die in der Forschung zur Differenzbildung zwischen Hamann und Jean Paul führte, mündet in einer gemeinsam geteilten Performanz des sich selbst negierenden Humors. Indem diese Zitate nicht mehr ,belegen', sondern sich selbst dekonstruieren, verfahren sie analog zur Selbstdekonstruktion Gottes am Kreuz, und lassen die Idole, die falschen Meinungen über die Natur, hinter sich, um die Natur selbst als den einen Kommentar des Wortes Gottes allererst sprechen zu lassen.

Eine solche Konstruktion tätigt Hamann zu Beginn von Kleeblatt Hellenistischer Briefe, wenn er als Hohepriester des Witzes ausgerechnet den Römerbrief $(\mathrm{XV}, 15)$ mit einem Zitat aus Lukrez kopuliert, das ihm diametral widerspricht. Im Kleeblatt Hellenistischer Briefe verzerrt Hamann Blaise Pascal in einer Zitation so, dass dadurch die eigentümliche Intention Pascals gerade zur Geltung kommt: „ich habe im Pascal einen Einfall über die Sprachen gefunden, von dem

54 Vgl. Christian Sinn: Jean Paul. Hinführung zu seiner Semiologie der Wissenschaft. Stuttgart 1995. 
ich mich wundere, dass er noch nicht belangt worden. Er hält alle Sprachen möglich zu entziffern (so viel mir mein Gedächtnis sagt); weil sie sich nämlich wie eine verborgene Schrift zur andern verhalten. Dass ein in der Mathematick geübter Kopf einen so offenbaren Trugschluss begehen können, ist leicht $\mathrm{zu}$ begreifen, wenn man nicht die Schwäche der menschlichen Erkenntnis zu einem blossen loci communi oder Schlupfwinkel seiner Sophistereyen macht. Aus seinem Satz, falls ich ihn recht behalten oder verstanden habe, folgt gerade das Gegenteil. ${ }^{\text {c5 }}$

Diese Stelle gibt einige Fragen auf. Entweder hat sich Hamann schlicht geirrt, denn sein Argument ist logisch gesehen falsch. Oder es handelt sich um eine Fehlschreibung, ein , nicht' wurde von Hamann vergessen. Das ist zwar rein spekulativ, aber möglich, zumal es sich um einen Text handelt, der ursprünglich nicht zur Veröffentlichung gedacht war. Drittens aber, und das ist meine hier vorgeschlagene Lesart, könnte sich auch um ein subtiles Argument handeln, das sich aus dem Kontext begründen lässt. Der erste Brief entwickelt nämlich das Argument, das wir Sprache nicht als reinen Algorithmus verstehen können, da sich das ,Schema' der Sprachen historisch wandelt und kontextuell definiert ist. Hamann macht das an folgendem Beispiel klar: Das arabische Wort ,Salamalec ${ }^{c}$ bedeute ,Friede sei mit Dir!', während im Französischen ,faire un grand Salamalec' hingegen, einen tiefen Bückling machen' bedeute. Selbst wenn sich die Araber bei ihrem Gruss mit einem tiefen Bückling verbeugten, so ist es doch unmöglich, aus dem arabischen Wort und Gruss den französischen Bückling (einer bestimmten Hofkultur) oder aus dem französischen Bückling den arabischen Gruss (einer bestimmten orientalischen Kultur) zu verstehen. Man braucht den jeweiligen pragmatisch-historischen Kontext einer Sprache und darin unterscheidet sich Sprache von mathematischen Zeichenbildungen.

Stellten wir uns eine philosophische Idealsprache nach dem Programm des Descartes vor, so gäbe es wortwörtlich nichts mehr zu sagen, denn alles wäre in ihr klar und deutlich geworden. Das ist der performative Widerspruch bei Descartes, den Hamann irrtümlich (?) Pascal unterstellt. Jedoch setzt bei Descartes und nicht bei Pascal der Mensch das, was er kann oder könnte, an die Stelle Gottes. Das wird am Begriff der Maschine deutlich. Dieser Begriff ist bei Descartes Ausdruck der höchsten menschlichen Konstruktionsleistung: Die Maschine lässt jene teleologischen Deutungen zu, die Descartes der Natur gerade abspricht. Damit kann bei diesem Philosophen Natur von vornherein nicht zum direkten Kommentar des Wortes Gottes werden wie bei Hamann, aber auch bei Jean Paul. Impliziert die Buch-Metapher den Menschen als Partner eines Autors, so gliedert die Maschinen-Metapher den Menschen als Element in ihren Funktionszusammenhang ein. Der Philosoph bestimmt sich in der französischen 
Aufklärung gerade dadurch, dass er wie alle anderen Menschen nur eine Maschine ist, die sich ab und zu selbst aufzuziehen weiß. Diese Selbstdefinition der Philosophie durch die Metaphysik der Maschine entbehrt allerdings nicht der Komik und regte dementsprechend die frühen Satiren Jean Pauls als Grundlage seiner späteren Werke an. ${ }^{56}$

Pascal aber beschreibt jene menschliche Misere, mit deren langanhaltenden und nicht einfach aufzulösenden Folgen wir immer noch nicht gelernt haben, gut umzugehen. „Der Mensch weiss nicht, welchen Rang er sich zuerkennen soll. Sichtbar ist er verwirrt von dem wahren Ort gefallen, ohne dass er ihn wiederfinden könnte. Unaufhörlich sucht er ihn in den undurchdringlichsten Finsternissen voller Unruhe und ohne Erfolg“. ${ }^{57}$

Pascal diagnostiziert selbstkritisch, wie sich der Mensch durch Zerstreuungen selbst ausbeutet, bis er zusammenbricht, um nur nicht die Verantwortung übernehmen zu müssen, die ihm durch Gott doch als ewige Zukunft bereits gegeben ist „Sollte die königliche Würde nicht an sich bedeutend genug sein, um den, der sie hat [...] glücklich zu machen?“58

Hamann spricht genau in diesem Sinne in seiner Auslegung des biblischen Herrschaftsauftrages über die Mitschöpfung von „der richterlichen und obrigkeitlichen Würde“ des Menschen als eines "politischen Thiers“, ${ }^{99}$ das durch seine Sprache in die fremde Natur und die eigene Geschichte eingreift, eingesetzt durch die Anrede Gottes als „Lehnträger und Erben der durch das Wort seines Mundes fertigen Welt" ${ }^{60}$ Von dieser Anrede Gottes aus bestimmt sich die lutherische Sozialethik als der umgreifende Kontext Hamanns: In einer Welt, in der ihr Schöpfer schon gesprochen hat, bedarf es nicht der geradezu perfiden Leistungslogik der Moderne, in der Menschen sich ständig selbst überholen müssen und mit dem Erreichten einfach nicht zufrieden sein dürfen.

Zwar ist dieser Kontext lutherischer Sozialethik die Begründung für Hamanns Kritik der sprachhistorischen Vernunft und auch noch für ihre Rezeption durch Jean Paul. ${ }^{61}$ Wie bereits die Hamann-Rezeption Herders belegt, gerät die Sprache

56 Wilhelm Schmidt-Biggemann: Maschine und Teufel. Jean Pauls Jugendsatiren nach ihrer Modellgeschichte. Freiburg, München 1975.

57 Blaise Pascal: Über die Religion und andere Gegenstände. Übertragen und herausgegeben von Ewald Wasmuth. Heidelberg 1963, Nr. 427.

58 Ebd., Nr. 142.

59 N III, 37,24-27: „In Ansehung der Geselligkeit hält der weise Stagyrit den Menschen für neutral. Ich vermuthe daher, daß der wahre Charakter unsrer Natur in der richterlichen und obrigkeitlichen Würde eines politischen Thiers bestehe [...]“.

60 N III, 32,9-10.

61 Zur Hamann-Rezeption Jean Pauls vgl. folgende kleine Auswahl: Beilage zun Denkwürdigkeiten (1773): HKA (wie Anm. 35), Abt. IV, Bd. 3/2, S. 224,10-17†; Biblische Betrachtungen (1748): Abt. III, Bd. 4, S. 45,11†; Bolingbroke-Übersetzung (1774): Abt. III, Bd. 4, S. 81,12†; Briefe: Abt. III, Bd. 3, S. 544 (89); Abt. III, Bd. 6, S. 286,18; Abt. IV, Bd. 4, S. 180,12-20; 
als eigenständige Macht dann aber in Konkurrenz zu Geltungsansprüchen lutherischer Offenbarungstheologie, auch jenen, die Hamann selbst vertritt, musste der Mensch sich doch nach Herder die Sprache selbst erschaffen, um überleben zu können. Wenn der Ursprung der Sprache mithin menschlich und nicht göttlich ist, dann steht auch alle theologische Rede mit ihrem Anspruch, sich nicht selbst gemacht zu haben, unter dem Verdacht, nur Menschenwerk und Rhetorik zu sein. Gewiss betreiben solche Form von rhetorischer Nicht- und Antirhetorik auch die Philosophie und die Wissenschaften, aber diese behaupten auch nie, Rede von Gott her zu sein.

Das vorläufige Fazit meines Zugangs auf Hamanns Kritik der sprachhistorischen Vernunft und ihrer Rezeption durch Jean Paul bleibt deshalb ambivalent und ist doch aussichtsreich. Weit über den typologischen Auslegungsaspekt von Natur und Geschichte hinaus sind Hamann und Jean Paul in ihrer Fortführung des sermo humilis und der in 1 Korinther 14 erhobenen Forderung nach Glossolalie gerade in ihrer Religionskritik zutiefst, christliche' Autoren. Aber die Kritik der philosophischen durch die metaphorischen Mittel religiöser Sprache lässt auch die Theologie nicht unberührt: auch sie wird unweigerlich und unvermeidlich von jenem ,test by ridicule` erfasst, den Shaftesbury als Kriterium der Rationalität empfohlen hatte und der für beide Autoren zentral ist. Unter der ,normalen' Perspektive des, wirklichen' Lebens scheint sich diese Kritik der sprachhistorischen Vernunft zwar Probleme zu schaffen, um sie auf wiederum unverständliche Weise zu lösen. Doch ihr geht es ja gerade um die Anerkennung, dass der Mensch sich selbst nicht nur häufig das Unverständliche ist, sondern auch sein sollte.

Meine philologischen ,Kreuz-Züge' münden daher in folgender verknappter, doch hoffentlich verständlicher These: Erst der Verzicht auf die absoluten Ansprüche von Religion, Staat, Philosophie und Wissenschaft stellt die Voraussetzung für jenen Begriff von Literatur als Medium der sprachhistorischen Kritik der Vernunft dar, den Hamann und Jean Paul ursprünglich für die Zwecke menschlicher Selbstverständigung entwickelten. Vielleicht und hoffentlich ist von ihren philologischen Experimenten aus nicht nur ein neues Gespräch zwischen Theologen und Literaturwissenschaftlern möglich, sondern qua philo-

Dangueil-Übersetzung (1757): Abt. III, Bd. 3, S. 268,10†; Golgatha und Scheblimini (1784): Abt. III, Bd. 4, S. 95, 21; Abt. III, Bd. 7, S. 107,13†; Abt. IV, Bd. 3/1, S. 164,31-32†; Hierophantische Briefe: Exzerpt IIc-42-1811-1812-0237; Kreuzzüge des Philologen (1762): Abt. III, Bd. 4, S. 95,19; Abt. III, Bd. 8, S. 319,10; Neue Apologie des Buchstaben h (1773): Abt. III, Bd. 3, S. 131,14†; Hamanns Schriften: Abt. IV, Bd. 3/2, S. 224,10-17†; Abt. IV, Bd. 6, S. 294,32; Sibyllinische Blätter (1819): Abt. III, Bd. 7, S. 476 (181); Abt. IV, Bd. 7, S. 306,7-8; Sokratische Denkwürdigkeiten (1759): Abt. III, Bd. 3, S. 544 (89); Abt. III, Bd. 6, S. 286,18; Abt. IV, Bd. 3/1, S. 163,20† und S. 164,29-30†; Wolken. Ein Nachspiel ... (1761): Abt. III, Bd. 3, S. 544 (89); Abt. III, Bd. 6, S. 286,18; Abt. IV, Bd. 4, S. 179,11. 
sophischem Humor auch theoretische Umbrüche für die sogenannten exakten Wissenschaften denkbar und wünschenswert, dem Vogel Merops gleich, „welcher zwar dem Himmel den Schwanz zukehrt, aber doch in dieser Richtung in den Himmel auffliegt. “62 In diesem Vogelbild wird der Humor als Umkehrung und Fortführung der alten Theologie verstanden: Diese schaut aus einer überirdischen, zeitlosen Welt auf die irdische, geschichtliche Welt hinunter und begreift sie als nichtig. Das aber ist Hybris. Der Humor jedoch misst mit der als nichtig verstandenen Welt die unendliche aus und versucht mit der vorläufigen Geschichte der Menschheit zu ihrem endgültigen Ende zu kommen, was ihm freilich nie gelingen kann. Der Humor muss sich deshalb selbst negieren, und lacht dann vor allem über sich selbst mit jenem Lachen „,worin noch ein Schmerz und eine Größe ist“. ${ }^{63}$ Humor ist, wenn man trotzdem schreibt.

62 Jean Paul: Vorschule der Ästhetik (wie Anm. 24), S. 129.

63 Ebd. 